\title{
Comparison of The Possibilities of Application of Spectrum and Gaming Theories in Modelling Market Economy Negotiations
}

\author{
Kęstutis Peleckis ${ }^{1,{ }^{*}}$, Valentina Peleckiene ${ }^{2}$, Kęstutis Peleckis ${ }^{3}$ \\ ${ }^{1}$ Department of Economics Engineering, Faculty of Business Management, Vilnius Gediminas \\ Technical Uni-versity, Sauletekio al. 11, LT-10223 Vilnius, Lithuania \\ 2 Department of Management, Faculty of Business Management, Vilnius Gediminas Technical \\ University, Sauletekio al. 11, LT-10223 Vilnius, Lithuania \\ ${ }^{3}$ Department of Business Technologies and Entrepreneurship, Faculty of Business Management, \\ Vilnius Gediminas Technical University, Saulètekio al. 11, LT-10223 Vilnius, Lithuania
}

\begin{abstract}
.
Research background: In negotiations, in modelling decision-making at both the individual and market levels, it is important to understand and be able to assess important aspects of economic behaviour. The theory of spectrum is proposed to be applied in modelling decision making. The object of the research is the possibilities of application of spectrum and game theories. Classical economic theory does not define subjective parameters of economic behaviour, therefore, when modelling market negotiation strategies, it is difficult to identify and evaluate appropriate parameters of economic behaviour required for decision-making in market negotiations. The spectrum theory approach can be used to model the economy, both at the individual and market levels, which is especially relevant in international business negotiations, where the modelling of solutions and various operations presents opportunities to assess subjective parameters.

Purpose of the article: The aim of the paper is to investigate and compare the application of spectral decay and gambling theories in modelling market economy negotiations.

Research methods: Scientific literature analysis, comparative, logical analysis and synthesis, comparative and generalization methods, game theory, quantum cognition methods.

Findings \& Value added: The article examines the basic principles of behavioural economics: the functions of assessing the psychological value and uncertainty of monetary gain or loss, how both theories take these principles into account: game and spectrum.
\end{abstract}

Keywords: behavioral economics; negotiations; market; game theory; distorted market

JEL Classification: $D 4 ; D 9 ; F 51$

\footnotetext{
*Corresponding author: k.peleckis@vgtu.lt
} 


\section{Introduction}

In negotiation, when modelling decision-making at both the individual and market levels, it is important to understand and be able to evaluate important aspects of economic behaviour. Therefore, the paper examines the basic principles of behavioural economics: the psychological value of monetary profit or loss and the functions of assessing uncertainty. How both theories take into account these principles: gambling and spectrum. The approach of spectrum theory can be used to model the economy, both at the individual and market levels.

The aim of the work is to investigate and compare the application of spectrum and game theories in modelling market economy negotiations.

The object of research is the application of spectrum and game theories.

Scientific problem - classical economic theory does not define subjective parameters of economic behaviour, therefore, when modelling market negotiation strategies, it is difficult to identify and evaluate appropriate parameters of economic behaviour required for decisionmaking in market negotiations.

Research methods - scientific literature analysis, comparative, logical analysis and synthesis, comparative and generalization methods, game theory, quantum cognition methods.

\section{Methods used in modelling market economy negotiations}

Game theory in economics is used as standard model for analysis of situations, on which people are interrelated $[1,2]$. For example, in negotiations, in the markets, where social problems are solved, such as selling or changing products and services. The problem of negotiations is reflected in situations, where subjects of business are trying to get profit in the market from possible situations [2-4]. In cases where monetary profit and losses compensate each other, so the usefulness of such profit and losses not necessarily offset each other, for example, if one of the individuals avoids the loss. The weakness in games of theoretical negotiation models is a high level of abstraction. It means that it is a lack of many aspects of negotiations. There are left many communication details in non-cooperative game theory, for example to persuade other part, to bluff and misrepresent interests $[3,5]$.

The models of negotiations foresee a high degree of negotiation effectiveness. Coase's theorem states that, assuming that there are no any transaction expenditures and that it is fully formed, the results of the negotiations are Pareto efficient. The development and using of Rational choice theory was important economic achievement $[4,6]$. A rational economic person, based on his beliefs, chooses an alternative that maximizes his choice, as it is given in the utility function, taking into account resource constraints such as money and time [5, 2]. In traditional Game theory a zero sum game describes the game, in which one player wins against other player, losing the net change of as-sets or benefits. The number of players is not limited. There is simplification, options and futures, which replicate bets on future stock prices, and profit is received when market prices fluctuate against expectations.

Information becomes the essential, if not the most important source [3, 4]. The scientists recently became increasingly aware that information pro-cessing is a physical phenomenon and that information theory is inseparable from both applied and basic physics. The attention for more physical information processing aspects revealed new perspectives on computational, cryptographic, and communication methods $[3,4]$. In many cases quantum description of the system gives advantages over the classical situation. It is similar that game theory, which studies (rational) decision-making in conflict situations, requires a quantum version - spectrum theory. 


\section{Opportunities to model market negotiations using classical Game theory}

Equations should be centred and should be numbered with the number on the right-hand side. The purpose of applying the Game theory is to determine economic behaviour, to find mathematically complete principles that would describe the rational behaviour of participants, and to determine the main characteristics of their behaviour [1-3, 4-7]. This is especially relevant in market economy negotiations in order to choose the most advantageous alternative for a market participant. The axiom of completeness states that the participant has clearly defined preferences and can always choose between the two alternatives. The axiom of transitivity assumes that if a participant prefers alternative $\alpha$ over $\beta$, and alternative $\beta$ over $\delta$, then he prefers alternative $\alpha$ over $\delta$. The interconnection axiom assumes that if a participant prefers the alternative to $\alpha$ rather than $\beta$, then the newly introduced unrelated alternative $\delta$ does not change that provision [1-3, 4-7].

The projected benefits of each alternative are described as the sum of all possible winnings when they are defined as coefficient. Suppose alternative $\alpha$ has two probable winnings: $\alpha_{1}$ size with possibility $\mathrm{P}\left(\alpha_{1}\right)$, and quantity $\alpha_{2}$ with possibility $\mathrm{P}\left(\alpha_{2}\right)$. Then probable winnings are

$$
U(\alpha)=P\left(\alpha_{1}\right) u\left(\alpha_{1}\right)+P\left(\alpha_{2}\right) u\left(\alpha_{2}\right)=P\left(\alpha_{1}\right) \alpha_{1}+P\left(\alpha_{2}\right) \alpha_{2}
$$

Game $\beta$ is more attractive if its expected utility satisfies $U(\beta)>U(\alpha)$.

Although the theory of expected utility is still the basis for the behaviour of many economic models, psychologists and economists have shown that the theory does not cover sequential cognitive phenomena [8-12]. One of the first attempts to modify the predicted utility theory was Perspective Theory, where solutions were analyzed, when there is a risk. This theory of expected utility has been modified in two ways. Firstly, it was said that in this case what matters are not the final amounts, but are the gains or losses, compared to a certain reference point. Second, it has been said that the results are evaluated by a nonlinear weighted uncertainty function rather than by probability itself. These two main findings of Perspective theory are illustrated in Figures 1 and 2.

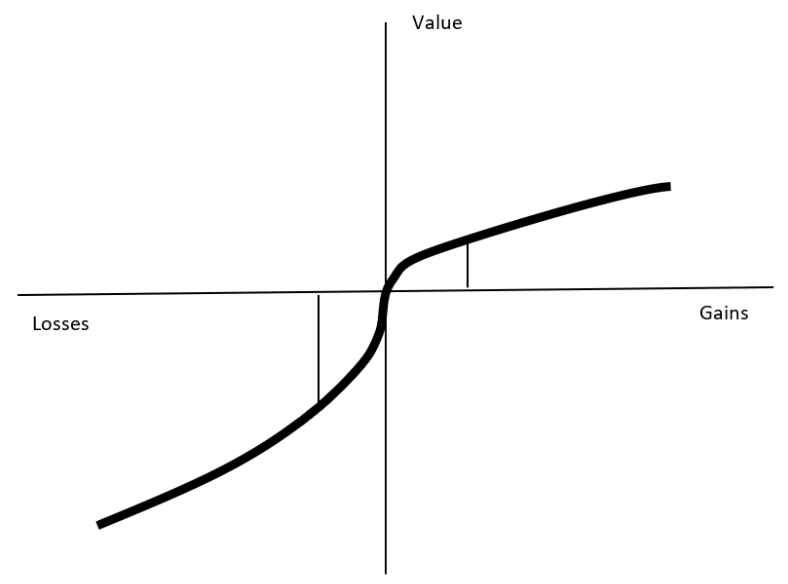

Fig. 1. A drawing of a value function showing the psychological value of an event. as a function of monetary gain or loss [15] 
The Center shall indicate the reference level at which the gain or loss is incurred. The function is rich because of the high profits or losses in addition, it is asymmetrical around a reference point, because a certain amount of loss is felt more than a comparable gain (dashed lines).

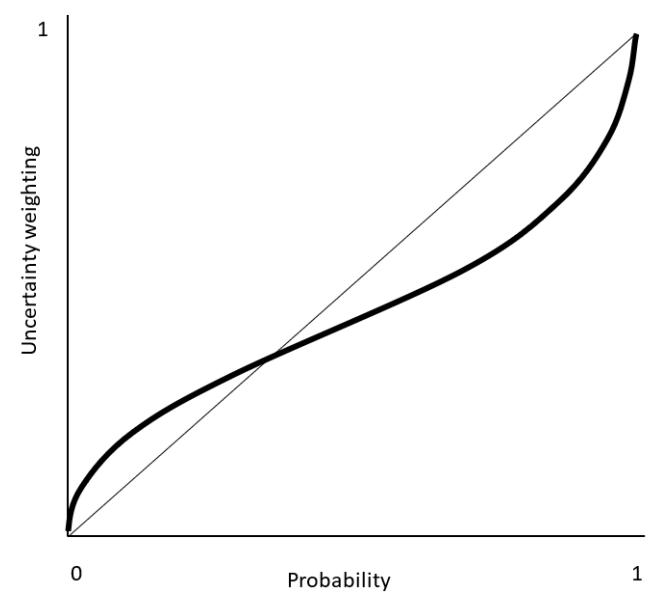

Fig. 2. Drawing of the uncertainty weighted function [15]

In the Theory of Expected Utility the event uncertainty factor is equal to its probability (dashed line). In Perspective theory, the curve is concave near 0 and convex at 1 (solid line). The curve will differ in terms of losses and gains, but the shapes will be similar. Considering the value function $\mathrm{v}(\mathrm{x})$. and the uncertainty estimation function $\mathrm{w}(\mathrm{p})$, the following formulas were formed:

$$
\begin{aligned}
& v(x)=-2(-x)^{0.5} \text { for } x<0 \\
& v(x)=x^{0.5} \text { for } x \geq 0
\end{aligned}
$$

And

$$
w(p)=\frac{p^{\gamma}}{\left(p^{\gamma}+(1-p)^{\gamma}\right)^{1 / \gamma}}
$$

Where $\gamma=0.61$.

These figures summarize together many basic cognitive phenomena that form the basis of behavioural economics. For example, losses and gains are felt in relation to a certain point of reference, which will depend on the context. This point is plotted on the horizontal axis of the value curve, which is equal to zero (shown in Figure 1). Most people don't seek a loss, but losing a certain amount is about twice as painful as winning the same amount. This is why the value curve is asymmetric around the initial position and the slope of the loss is steeper [5-7].

Experiments also show that we do not properly evaluate possible outcomes according to their probabilities. We change much more sensitively. probability from 0 to 0.1 , or from 0.9 to 1 . than from 0.4 to 0.5 . this is why the uncertainty function in Figure 2 is bent at zero and convex at 1.

The difference between Perspective Theory and Expected Benefit Theory. is shown instead of formula 


$$
U(\alpha)=p\left(\alpha_{1}\right) \alpha_{1}+p\left(\alpha_{2}\right) \alpha_{2}
$$

we are writing:

$$
U(\alpha)=w\left(\alpha_{1}\right) v\left(\alpha_{1}\right)+w\left(\alpha_{2}\right) v\left(\alpha_{2}\right)
$$

Where $\mathrm{v}$ is a function of value and $\mathrm{w}$ is a function of estimating uncertainty. Perspective theory can be seen as a modified version of the Expected Benefit Theory, where the linear relationship is replaced by nonlinear curves. It could be considered the Allais paradox:

Game $\alpha$ : choice between

$\alpha_{1} 500$ EUR with 80 percent. probability

$\alpha_{2} 375$ EUR with 100 percent. probability

Game $\beta$ : choice between

$\beta_{1}$ EUR 500 with $20 \%$. probability

$\beta_{2} 375$ EUR with 25 percent. Probability

According to the Theory of Expected Benefits, we have:

for game $\alpha$ :

$$
\begin{aligned}
& U\left(\alpha_{1}\right)=p\left(\alpha_{1}\right) \alpha_{1}=0.80 \times 500=400 \\
& U\left(\alpha_{2}\right)=p\left(\alpha_{2}\right) \alpha_{2}=1.00 \times 375=375
\end{aligned}
$$

For game $\beta$

$$
\begin{aligned}
& U\left(\beta_{1}\right)=p\left(\beta_{1}\right) \beta_{1}=0.20 \times 500=100 \\
& U\left(\beta_{2}\right)=p\left(\beta_{2}\right) \beta_{2}=0.25 \times 375=94
\end{aligned}
$$

In the first choice, the game offers slightly better benefits. In practice, however, participants choose the first option in the $\beta$ game and the second option in the $\alpha$ game. The reason that in game $\alpha$ is the warranty option, which is more attractive. However, this meant that their Utility Theory was inconsistent, which violated the Axioms of Proposed Utility Theory [7].

In Perspective Theory, using the value and uncertainty estimation function, these calculations become for $\alpha$ game:

$$
\begin{aligned}
& U\left(\alpha_{1}\right)=w\left(\alpha_{1}\right) v\left(\alpha_{1}\right)=0.61 * 22.36=13.64 \\
& U\left(\alpha_{2}\right)=w\left(\alpha_{2}\right) v\left(\alpha_{2}\right)=1.00 * 19.37=19.37
\end{aligned}
$$

For $\beta$ game:

$$
\begin{aligned}
& U\left(\beta_{1}\right)=w\left(\beta_{1}\right) v\left(\beta_{1}\right)=0.26 * 22.36=5.81 \\
& U\left(\beta_{2}\right)=w\left(\beta_{2}\right) v\left(\beta_{2}\right)=0.29 * 19.37=5.62
\end{aligned}
$$

The most attractive alternative is now $\alpha_{2}$ and $\beta_{1}$, which corresponds to the experiments. The reason for that was. psychological overestimation of the guarantee.

The Ellsberg paradox should also be examined. There is a box with 180 balls, of which 60 are red and 120 are black or yellow. There are two choices: in the case of $\alpha$, you can bet on red or black; In the case of $\beta$, you can bet on red or yellow, or black or yellow. In each game, the probability of finding a color is 1 to 3 . The difference is that in game $\beta$, each guess has a yellow color as an alternative. Therefore, if you want red in the case of $\alpha$, then you have 
to choose red or yellow in game $\beta$. Most people see it differently - they don't look at the color of the ball, but at the uncertainty [5-7].

In game $\alpha$ we know that number of red will be 60 and black is unknown. Therefore, the red color is chosen in the game $\alpha$. In the game $\beta$. the number of yellow balls is unknown, but the known amount is 120 . Therefore, black or yellow is chosen, again due to less uncertainty. This inconsistency contradicts the theory of Expected Utility, but it also denies the Theory of Prospects for the simple reason that probabilities are not known, making it impossible to reconcile them with the function of the uncertainty weight [9].

Using classical theory, there are a number of cognitive phenomena that are difficult to predict [11-12]. These include so-called merge and detachment effects, order effect, and preference change. These cases show that context and measurement procedures affect responses. The Elsberg paradox presents two formally identical variants, with a script difference. Uncertainty about black and yellow balls creates interference that determines the assessment. It will therefore be examined further. a quantum approach that could be used to address these paradoxes.

\section{Possibilities of market negotiation modelling using spectrum theory}

In this section, we will examine spectrum theory, and its applications for modelling market negotiations, with the aim to fill the limited possibilities of classical theory to evaluate subjective parameters of economic behaviour. Therefore, in the case of the Alay effect, we note that the problem can be presented in the form of a matrix, forming a benefit matrix for the game $\alpha$

$$
U=\left(\begin{array}{ccc}
u_{1} & 0 & 0 \\
0 & u_{2} & 0 \\
0 & 0 & u_{3}
\end{array}\right)=\left(\begin{array}{ccc}
500 & 0 & 0 \\
0 & 375 & 0 \\
0 & 0 & 0
\end{array}\right)
$$

The probabilities will be the vectors $\Psi_{1}=\left(\begin{array}{lll}\sqrt{0.8} & 0 & \sqrt{0.2}\end{array}\right)$ ir $\Psi_{2}=\left(\begin{array}{lll}0 & 1 & 0\end{array}\right)$, where the third component takes into account the possibility of not winning anything. The likely benefits in this case are

$$
\begin{gathered}
O(\Psi)=\Psi U \Psi^{T} \\
O\left(\Psi_{\alpha 1}\right)=\left(\begin{array}{lll}
\sqrt{0.8} & 0 & \sqrt{0.2}
\end{array}\right)\left(\begin{array}{ccc}
500 & 0 & 0 \\
0 & 375 & 0 \\
0 & 0 & 0
\end{array}\right)\left(\begin{array}{c}
\sqrt{0.8} \\
0 \\
\sqrt{0.2}
\end{array}\right)=400 \\
O\left(\Psi_{\alpha 2}\right)=\left(\begin{array}{lll}
0 & 1 & 0
\end{array}\right)\left(\begin{array}{ccc}
500 & 0 & 0 \\
0 & 375 & 0 \\
0 & 0 & 0
\end{array}\right)\left(\begin{array}{l}
0 \\
1 \\
0
\end{array}\right)=375 \\
O\left(\Psi_{\beta 1}\right)=\left(\begin{array}{lll}
\sqrt{0.2} & 0 & \sqrt{0.8}
\end{array}\right)\left(\begin{array}{ccc}
500 & 0 & 0 \\
0 & 375 & 0 \\
0 & 0 & 0
\end{array}\right)\left(\begin{array}{c}
\sqrt{0.2} \\
0 \\
\sqrt{0.8}
\end{array}\right)=100 \\
O\left(\Psi_{\beta 1}\right)=\left(\begin{array}{llll}
0 & \sqrt{0.25} & \sqrt{0.75}
\end{array}\right)\left(\begin{array}{ccc}
500 & 0 & 0 \\
0 & 375 & 0 \\
0 & 0 & 0
\end{array}\right)\left(\begin{array}{c}
0 \\
\sqrt{0.25} \\
\sqrt{0.75}
\end{array}\right)=94
\end{gathered}
$$

The answers are the same as before.

We can also use spectral decay to describe $U$ 


$$
O(\Psi)=\Psi U \Psi^{T}=\Psi\left(\sum_{i} u_{i} V_{i} V_{i}^{T}\right) \Psi^{T}=\sum_{i} u_{i}\left|\Psi V_{i}\right|^{2}
$$

Where $V_{i}$ is the real vector of column 0 with respect to the real value $U i$, and ViViT is the projection operator is the projection operator that protrudes into that self-vector. In the classical case, where 0 is diagonal, the matrix $\mathrm{V}$ of the real vector is an identical matrix, the formula decreases to the weighted expected benefit function, where the win $u_{i}$ is weighted by the possibility

In this case we can write:

$$
\left|\Psi V_{i}\right|^{2}=\Psi_{i}^{2}
$$

$$
O(\Psi)=500 \Psi\left(\begin{array}{lll}
1 & 0 & 0 \\
0 & 0 & 0 \\
0 & 0 & 0
\end{array}\right) \Psi^{T}+375 \Psi\left(\begin{array}{lll}
0 & 0 & 0 \\
0 & 1 & 0 \\
0 & 0 & 0
\end{array}\right) \Psi^{T}+0 \Psi\left(\begin{array}{lll}
0 & 0 & 0 \\
0 & 0 & 0 \\
0 & 0 & 1
\end{array}\right) \Psi^{T}
$$

In the quantum case, the matrix $U$ responds to the observation, which is a Hemitian operator with real true values. Expected benefit $\mathrm{O}(\Psi)$ is the probable value of the observation when the system is in place

$$
|\Psi\rangle=\sum_{i}\left\langle V_{i} \mid \Psi\right\rangle *\left|V_{i}\right\rangle
$$

Where the weights $\left\langle V_{i} \mid \Psi\right\rangle^{2}$ take into account the conditions $\left|\Psi V_{i}\right|^{2}$ in the matrix version, and show the subjective weight assigned to the win Ui. In other words, we break down $\Psi$ the position of the decision maker to the real vectors $V_{i}$ where each takes into account the corresponding result, and uses the result to reach the observed $\mathrm{O}(\Psi)$.

In the classic case, probabilities $\Psi$ indicate the objective state of the game, and the weights combine these probabilities with the winners $V_{i}$. The problem is that there is no punishment administered to the fact that nothing is unwinnable, what is known as influencing decisions [13-16]. Based on La Mura's theory of projected expected benefits, this can be done in a quantum system by choosing an observational $U$ that would be a Hermitian operator where non-diagonal conditions are not zero. In this case, the weights $\left\langle V_{i} \mid \Psi\right\rangle^{2}$ will not only weigh the result according to its probability of occurrence, but may include other factors as the fact that the appearing result means that the next result will not occur. For example, a low degree of fear risk of not winning anything (the third component) can be modeled by including

Which gives

$$
\mathrm{U}=\left(\begin{array}{ccc}
500 & 0 & -40 \\
0 & 375 & -40 \\
-40 & -40 & 0
\end{array}\right)
$$

$$
\begin{array}{r}
O\left(\Psi_{\alpha 1}\right)=\left(\begin{array}{lll}
\sqrt{0.8} & 0 & \sqrt{0.2}
\end{array}\right)\left(\begin{array}{ccc}
500 & 0 & -40 \\
0 & 375 & -40 \\
-40 & -40 & 0
\end{array}\right)\left(\begin{array}{c}
\sqrt{0.8} \\
0 \\
\sqrt{0.2}
\end{array}\right)=368 \\
O\left(\Psi_{\alpha 2}\right)=\left(\begin{array}{lll}
0 & 1 & 0
\end{array}\right)\left(\begin{array}{ccc}
500 & 0 & -40 \\
0 & 375 & -40 \\
-40 & -40 & 0
\end{array}\right)\left(\begin{array}{l}
0 \\
1 \\
0
\end{array}\right)=375 \\
O\left(\Psi_{\beta 1}\right)=\left(\begin{array}{lll}
\sqrt{0.2} & 0 & \sqrt{0.8}
\end{array}\right)\left(\begin{array}{ccc}
500 & 0 & -40 \\
0 & 375 & -40 \\
-40 & -40 & 0
\end{array}\right)\left(\begin{array}{c}
\sqrt{0.2} \\
0 \\
\sqrt{0.8}
\end{array}\right)=68 \\
O\left(\Psi_{\beta 1}\right)=\left(\begin{array}{llll}
0 & \sqrt{0.25} & \sqrt{0.75}
\end{array}\right)\left(\begin{array}{ccc}
500 & 0 & -40 \\
0 & 375 & -40 \\
-40 & -40 & 0
\end{array}\right)\left(\begin{array}{c}
0 \\
\sqrt{0.25} \\
\sqrt{0.75}
\end{array}\right)=59,10
\end{array}
$$


Therefore, the alternatives $\alpha_{2}$ and $\beta_{1}$ are now chosen as more useful.

In summary, the diagonal utility matrix means that perspectives are evaluated by projecting the results of an underlying object with known benefits [17-22]. In practice, the person will appreciate the perspective by designing on subjective frameworks that will not fit perfectly with the classical version and will give negative weights to undesirable outcomes [23-25]. This method transfers non-zero non-diagonal data to the U utility matrix, which has the advantage of simplicity, of course $U$ has two negative effects: objective and subjective weights. Therefore, instead of calculating $U$, the alternative method provides a subjective basis. Which projects the position $\Psi$ and uses the spectral decay together with the normalized conditions to generate the $\mathrm{U}$ result.

\section{Conclusions}

In market economy negotiations, the participants are confronted with vast amounts of information about the objective parameters that determine the context of the negotiations. However, no less important is subjective economic behaviour, which is not always possible to assess, and existing theories have not considered subjectivity as an important aspect of economic behaviour. The assessment and formation of bargaining power is especially relevant in negotiations, where it is necessary to harmonize the processes of interaction between participants of different cultures, assessing the con-text of negotiations, cultural differences, aspects of conflict prevention. The assessment of these aspects of internationalization is relevant in order to make more effective use of the potential of bargaining power in market economy negotiations. This highlights the need to look for modelling approaches focused on cross-cultural compatibility, conflict prevention, and a more effective cognition of negotiation context.

The Spectrum theory approach can be used to model economic behaviour, more specifically negotiation strategies, at both the individual and market levels. This is especially relevant in international business negotiations, when modelling solutions and various operations, there are opportunities to evaluate subjective parameters. Classical economic theory does not define subjective parameters of economic behaviour, therefore, when modelling market negotiation strategies, it is difficult to identify and evaluate appropriate parameters of economic behaviour required for decision-making in market negotiations. Therefore, the possibilities of application of Spectrum theory in comparison with the classical theory are examined in this work. Spectrum theory is proposed to be applied in modelling decision making. Using classical theory, there are a number of cognitive phenomena that are difficult to predict. These include so-called merge and detachment effects, order effect, and preference change. These cases show that context and measurement procedures affect responses.

In summary, the diagonal utility matrix means that perspectives are valued in design on a basis, having object results with known benefits. In practice, the person will appreciate the perspective by designing on subjective frameworks that will not fit perfectly with the classical version and will give negative weights to undesirable out-comes. This method transfers non-zero non-diagonal data to the U utility matrix, which has the advantage of simplicity Of course $U$ has two negative effects: objective and subjective weights. Therefore, instead of calculating $U$, the alternative method provides a subjective basis, which projects the position $\Psi$ and uses the spectral decay together with the normalized conditions to generate the $\mathrm{U}$ result. 


\section{References}

1. Palafox-Alcantar, P. G., Hunt, D. V. L., \& Rogers, C. D. F. (2020). The complementary use of game theory for the circular economy: A review of waste management decisionmaking methods in civil engineering. Waste Management, 102, 598-612.

2. Piotrowski, E. W., \& Sładkowski, J. (2003). Quantum english auctions. Physica A: Statistical Mechanics and its Applications, 318(3-4), 505-515.

3. Omrani, H., Fahimi, P., \& Mahmoodi, A. (2020). A data envelopment analysis game theory approach for constructing composite indicator: An application to find out development degree of cities in West Azarbaijan province of Iran. Socio-Economic Planning Sciences, 69, 100675.

4. Becchetti, L., Bruni, L., \& Zamagni, S. (2019). The Microeconomics of Wellbeing and Sustainability: Recasting the Economic Process. Academic Press.

5. Golroudbary, S. R., El Wali, M., \& Kraslawski, A. (2020). Rationality of using phosphorus primary and secondary sources in circular economy: Game-theory-based analysis. Environmental Science \& Policy, 106, 166-176.

6. Whalen, K. A., Berlin, C., Ekberg, J., Barletta, I., \& Hammersberg, P. (2018). 'All they do is win': Lessons learned from use of a serious game for Circular Economy education. Resources, Conservation and Recycling, 135, 335-345.

7. Liu, G., Xiao, Z., Tan, G., Li, K., \& Chronopoulos, A. T. (2020). Game theory-based optimization of distributed idle computing resources in cloud environments. Theoretical Computer Science, 806, 468-488.

8. de Lange, D., \& Valliere, D. (2020). Investor preferences between the sharing economy and incumbent firms. Journal of Business Research, 116, 37-47.

9. Skare, M., \& Porada-Rochoń, M. (2020). Multi-channel singular-spectrum analysis of financial cycles in ten developed economies for 1970-2018. Journal of Business Research, 112, 567-575.

10. Gazda, J., Bugar, G., Volosin, M., Drotar, P., Horvath, D., \& Gazda, V. (2017). Dynamic spectrum leasing and retail pricing using an experimental economy. Computer Networks, 121, 173-184.

11. Suzanne, E., Absi, N., \& Borodin, V. (2020). Towards Circular Economy in Production Planning: Challenges and Opportunities. European Journal of Operational Research, 287(1), 168-190.

12. Fahrenberg, U., \& Legay, A. (2020). A linear-time-branching-time spectrum for behavioral specification theories. Journal of Logical and Algebraic Methods in Programming, 110, 100499.

13. Riser, R., Osipov, V. A., \& Kanzieper, E. (2020). Nonperturbative theory of power spectrum in complex systems. Annals of Physics, 413, 168065.

14. Orrell, D. (2020). A quantum model of supply and demand. Physica A: statistical Mechanics and its Applications, 539, 122928.

15. Shubik, M. (1999). Quantum economics, uncertainty and the optimal grid size. Economics Letters, 64(3), 277-278.

16. Rashkovskiy, S., \& Khrennikov, A. (2020). Psychological 'double-slit experiment'in decision making: Quantum versus classical. Biosystems, 195, 104171.

17. Samadi, A. H., Montakhab, A., Marzban, H., \& Owjimehr, S. (2018). Quantum BarroGordon game in monetary economics. Physica A: Statistical Mechanics and its Applications, 489, 94-101. 
18. Phoenix, S., Khan, F., \& Teklu, B. (2020). Preferences in quantum games. Physics Letters A, 384(15), 126299.

19. Wang, H., \& Xiang, H. (2019). Quantum algorithm for total least squares data fitting. Physics Letters A, 383(19), 2235-2240.

20. Asano, M., Basieva, I., Khrennikov, A., Ohya, M., \& Tanaka, Y. (2012). Quantum-like dynamics of decision-making. Physica A: Statistical Mechanics and its Applications, 391(5), 2083-2099.

21. Piotrowski, E. W., \& Sładkowski, J. (2002). Quantum market games. Physica A: Statistical Mechanics and its Applications, 312(1-2), 208-216.

22. Danilov, V. I., \& Lambert-Mogiliansky, A. (2018). Preparing a (quantum) belief system. Theoretical Computer Science, 752, 97-103.

23. Moreira, C., Tiwari, P., Pandey, H. M., Bruza, P., \& Wichert, A. (2020). Quantum-like influence diagrams for decision-making. Neural Networks, 132, 190-210.

24. Haven, E., \& Khrennikov, A. (2017). The use of action functionals within the quantumlike paradigm. Journal of Mathematical Psychology, 78, 13-23.

25. Yang, Z., \& Zhang, X. (2019). Quantum repeated games with continuous-variable strategies. Physics Letters A, 383(24), 2874-2877. 